\title{
X-ray study of W/Si multilayers for the HEFT hard $x$-ray telescope
}

Kristin K. Madsen, Finn E. Christensen, Carsten P. Jensen, Eric Ziegler, William W. Craig, et al.

Kristin K. Madsen, Finn E. Christensen, Carsten P. Jensen, Eric Ziegler, William W. Craig, Kurt S. Gunderson, Jason E. Koglin, K. Pedersen, "X-ray study of W/Si multilayers for the HEFT hard x-ray telescope," Proc. SPIE 5168, Optics for EUV, X-Ray, and Gamma-Ray Astronomy, (29 January 2004); doi: 10.1117/12.505665

SPIE Event: Optical Science and Technology, SPIE's 48th Annual Meeting, 2003, San Diego, California, United States 


\title{
X-ray study of W/Si multilayers for the HEFT hard x-ray telescope
}

\author{
K. K. Madsen ${ }^{a}$, F. E. Christensen ${ }^{a}$, C. P. Jensen ${ }^{a}$, E. Ziegler ${ }^{b}$, W. W. Craig ${ }^{c}$, K. Gunderson ${ }^{c}$, \\ J. E. Koglin ${ }^{d}$, K. Pedersen ${ }^{e}$ \\ ${ }^{a}$ Danish Space Research Institute, Copenhagen, Denmark \\ ${ }^{b}$ European Synchrotron Radiation Facility, Grenoble, France \\ ${ }^{c}$ Lawrence Livermore National Laboratory, California \\ ${ }^{d}$ Columbia University, New York \\ ${ }^{e}$ Astronomical Observatory, Copenhagen, Denmark
}

\begin{abstract}
This paper outlines an in-depth study of the W/Si coated mirrors for the High Energy Focussing Telescope (HEFT). We present data taken at 8, 40 and $60 \mathrm{keV}$ obtained at the Danish Space Research Institute and the European Synchrotron Radiation Facility in Grenoble. The set of samples were chosen to cover the parameter space of sample type, sample size and coating type. The investigation includes a study of the interfacial roughness across the sample surface, as substrates and later as coated, and an analysis of the roughness correlation in the $\mathrm{W} / \mathrm{Si}$ coatings for $\mathrm{N}=10$ deposited bilayers. The powerlaw graded flight coating for the HEFT mirrors is studied for uniformity and scatter, as well as its performance at high energies.
\end{abstract}

\section{INTRODUCTION}

A great concern in the contruction of any imaging device is the presence of imperfections that may result in a severe degrading of the Point Spread Function of an image. While this causes optical telescopes some problems, for an x-ray telescope it becomes much greater. The requirements for multilayers to efficiently reflect x-rays are therefore very stringent and tolerances of deviations very small. In a mission like HEFT, which requires the assembly of more than 1400 multilayered mirror segments per telescope, it is thus of great importance that the average quality of its mirrors are within the set requirements. In this experiment we set out to test this quality through a careful investigation of the roughness and interfacial diffusion, and analysis of the scattered diffuse component.

The first section summarizes the theory of reflectivity and scattering, followed by a brief description of the HEFT optic and the graded W/Si coating recipe used on the mirrors. Section 3 outlines the experiment and the sample set used in the study. Section 4 then presents $8 \mathrm{keV}$ data obtained at the DSRI, and section 5 data at $40 \mathrm{keV}$ and $60 \mathrm{keV}$ obtained at the ESRF. Finally we summarize our findings.

\section{THEORY}

Scattering from a surface with interfacial width, $\sigma$, can be divided into two distinct components: the specular reflectivity in which the momentum is conserved within the plane of the surface and the reflectivity is confined to a 1-dimensional sheet, and non-specular reflectivity, which is the scattered radiation exiting at all other angles. The scattering from an ideal surface is exactly described by the Fresnel equations ${ }^{1}$ and does not include a nonspecular diffuse component. The effect of an interfacial roughness is to remove part of the specular intensity from an ideal surface and redistribute it at other scattering angles. This distribution of scattered intensity will therefore be intrinsically linked to the type of interfacial roughness that caused the scatter. The width of an interface, $\sigma$, is given by

$$
\sigma^{2}=\int z^{2} p^{\prime}(z) d z
$$

where $z$ is the coordinate perpendicular to the surface and $p(z)$ the interfacial profile between two layers. Different types of roughness are given by different types of interfacial profiles and two very commonly used are: the classical 
Table 1. 2 examples of a HEFT1 recipe

\begin{tabular}{|c|c|c|c|c|c|}
\hline Multilayer Group & Layers & $\mathrm{d}_{\min }(\AA)$ & $\mathrm{d}_{\max }(\AA)$ & $N_{\text {bilayer }}$ & $\mathrm{c}$ \\
\hline 1 & L1-L6 & 33.3 & 297.6 & 125 & 0.236 \\
5 & L26-L32 & 26.1 & 191.8 & 250 & 0.225 \\
10 & L64-L72 & 23.0 & 110.7 & 312 & 0.195 \\
\hline
\end{tabular}

diffusion between two materials and roughness from a real sharp interfacial height variation. The former causes a grading of the composition through the interface and is best modelled by an error-function, while the latter can be described by an exponentional function, although this is by no means the only possibility. ${ }^{2}$ Real surfaces are considered to be a combination of both types, that is $\sigma_{=} \sqrt{\sigma_{d}^{2}+\sigma_{r}^{2}}$, where $\sigma_{d}$ is the interface width due to diffusion and $\sigma_{r}$ due to roughness.

The specular amplitude reflectivity is calculated by modifying the Fresnel equations for an ideal interface with an exponential term

$$
\tilde{r_{0}}=r_{0} \exp \left(-2 k_{0} k_{1} \sigma^{2}\right)
$$

$k_{0}$ is the perpendicular component of the incident wave vector, $k_{1}$ the same component of the refracted wave and $r_{0}$ the reflected amplitude from an ideal surface. This expression was derived by Nèvot and Croce ${ }^{3}$ and considered valid if the correlation length, $\xi$, is short so that $\xi k_{0}^{2} /|\mathbf{k}| \ll 1$.

From the above expression it is obvious that the specular reflectivity does not distinguish between roughness types, and therefore in order to learn anything about the origin of the roughness, it is necessary to also look at the non-specular scattering. It is well described by the Distorted Wave Born Approximation scalar theory, which treats the roughness as a pertubation of the reflectivity of an ideal surface. An important factor of this scattering, is the manner in which the roughness is propagating through the multilayer stack. A roughness which is completely uncorrelated will distribute the non-specular reflectivity in a uniform halo, while a perfectly correlated roughness will behave like a crystal lattice and arrange the scatter into 'streaks' obeying the Braggcondition. ${ }^{4}$ This latter kind of roughness is known as $\sigma_{\text {corr }}$. The real roughness $\sigma_{r}$ can be described as a sum of correlated and uncorrelated roughness $\sigma_{r}^{2}=\sigma_{r, \text { corr }}^{2}+\sigma_{r, \text { uncorr }}^{2}$. At the specular condition the reflectivity is in part biased by the scattered uncorrelated intensity which happens to exit at the specular condition, and to obtain the true interfacial width, $\sigma$, it is therefore necessary to first subtract the uncorrelated intensity. The correlated roughness, $\sigma_{r, \text { corr }}$, can be found by fitting the streaks, and thus a measure of the diffuse interfacial width can be found from $\sigma_{d}=\sqrt{\sigma^{2}-\sigma_{r}}$. This assumes that the uncorrelated component is negligible, which is a fairly good assumption when the Bragg peaks in the off-specular scan are of the same width as the specular.

One way to describe a rough surface is by assuming it is self-affine, as Sinha $e t a l^{5}$ have done, and in that case it can be assigned a height-height correlation function of the type

$$
<h(r-R) h(r)>=\sigma_{\text {corr }}^{2} \exp \left[-(|R| / \xi)^{2 / h}\right]
$$

The fractal dimension is $D=3-h$ where $0<h \leq 1$ and $\xi$ is the in-plane correlation length. In the event that the roughness is perfectly correlated it will be the same for all layers, while a roughness that is uncorrelated will at each interface be described by its own separate correlation length. The fractal exponent $h$ describes how rugged the surface is and for $h \rightarrow 0$ it becomes increasingly more jagged, while for $h=1$ the function becomes a Gaussian distribution.

\subsection{The HEFT mirrors}

The High Energy Focussing Telescope is a grazing incidence conical approximation to a Wolter-I optic consisting of a staggering 1440 mirror segments arranged in 72 concentrically aligned shells with radii ranging from 40-120 $\mathrm{mm}$. The length of the telescope is $400 \mathrm{~mm}$ which is divided into two $200 \mathrm{~mm}$ sections: the upper conical parabola approximation and the lower conical hyperbola approximation. Since one mirror segment is $100 \mathrm{~mm}$ long and curved to cover an angle of 72 degrees, one shell needs twenty pieces, ten for the lower and ten for the upper. 


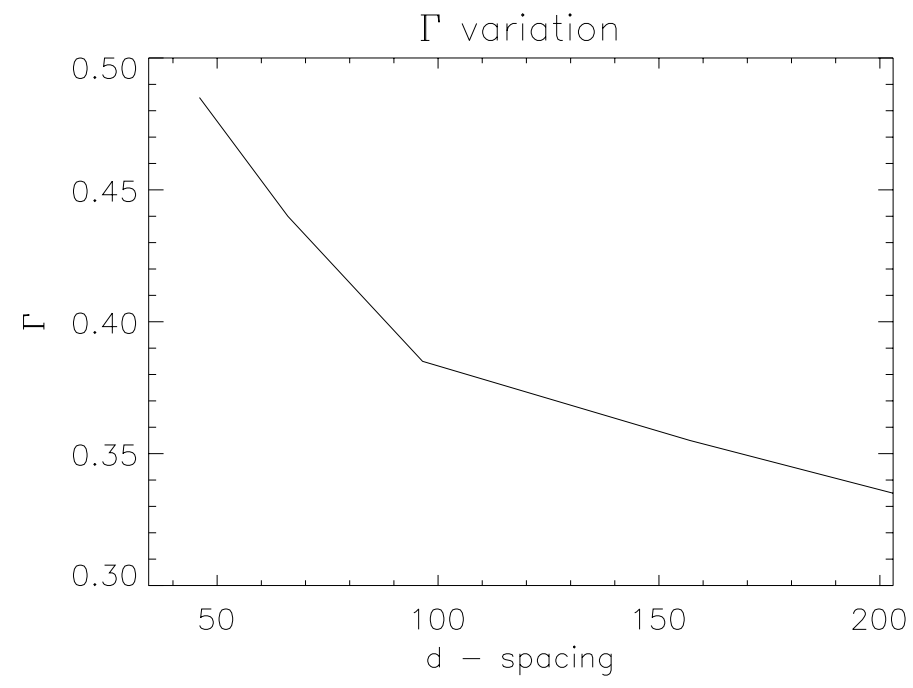

Figure 1. $\Gamma$ variation versus period.

A multilayer coating with a constant bilayer thickness, $d$, is characterized by the number of bilayers, $N, d$, and the fraction of the tungsten thickness, $d_{W}$, to the total thickness, $\Gamma=d_{W} / d$. HEFT uses a W/Si multilayer coating with an in-depth graded period to extend the energy range to $70 \mathrm{keV}$. The variation in thickness spacing, $d$, is designed as a powerlaw

$$
d_{i}=\frac{a}{(b+i)^{c}}, \quad i=1, \ldots, N
$$

where $a, b$ and $c$ are constants of the powerlaw model, and $i$ is the bilayer number, with bilayer $\mathrm{N}$ being right next to the substrate. The period $d$ thus varies from a $d_{\min }$ value at the substrate increasing up through the stack until it reaches its maximum value $d_{\max }$ at the top layer. This ensures that the hardest x-rays are reflected from the bottom of the stack and the softer x-rays from the top of the stack. Once the parameter $c$ has been determined, $a$ and $b$ are uniquely determined by $d_{\max }$ and $d_{\min }$. Optimization of the coating recipe was therefore performed across the phase space $d_{\max }, d_{\min }, N_{\text {bilayer }}, c$ and $\Gamma$. To make optimizations more accessible the mirrors were divided into 10 groups according to range of their on-axis grazing incidence angle. ${ }^{6}$ Examples of recipes 1,5 and 10 are given in table 1 . In our optimization $\Gamma$ was for simplicity assumed to be a constant, but in reality it is a depth graded function, as can be seen from figure 1, which shows a calibration measurement of the DSRI multilayer coating facility. ${ }^{7}$ All mirror groups follow the same $\Gamma$-profile and the models used to fit the data are able to take this variation into account.

The above described coating has been dubbed a 'flight' coating, and it will hereafter be referred to as such. Mirror segments are named according to their intended placement on the telescope, so that L72 is a segment of layer 72 .

\section{EXPERIMENTS}

The objective of the study was to characterize as many surface properties as possible over the entire surface of the mirror segments and through the entire process, from before slumping of the mirror to after its coating. To achieve this the samples were measured, when possible, both before and after their coating.

Three different types of scans were performed. An in-plane position sensitive detector was used to produce a 2dimensional map in angle space $\left(\theta_{\text {inci }}, \theta_{\text {scat }}\right)$. From this essentially all other scans can be extracted, and the entire diffuse reflectivity structure is also revealed. Supplementing this mesurement we used standard $(\theta, 2 \theta)$ specular reflectivity scans and transverse $\omega$ scans, which holds the $2 \theta$ detector angle constant while the sample holder is scanned from 0 to $2 \theta$. A fourth 'off-set' specular scan was extracted from the map off-set with $\theta_{\text {scat }}=0.2^{\circ}$. 


\subsection{Samples}

To ensure that the study covered as many parameters as possible it was decided to vary the following:

- Energy. Measurements at $8 \mathrm{keV}$ were performed at the Danish Space Research Institute, and the high energies 40 and $60 \mathrm{keV}$ at the European Synchrotron Radiation Facility in Grenoble.

- Radius of the mirrors. The choice fell on 3 different radii; small radii at $45 \mathrm{~mm}$ (layers $3-5$ ), intermediate radii at $70 \mathrm{~mm}$ (layers 30-32) and large radii at $115 \mathrm{~mm}$ (layers $71-72$ ).

- Measurement points across the mirrors. 9 points were deemed sufficient to map the surface. The geometry of a curved mirror is given relative to its central point $(\psi, l)=\left(0^{\circ}, 0 \mathrm{~mm}\right)$ so that a point on the surface shifted towards the x-ray source at constant azimuthal angle is positive. The points used are; $(\psi, l)=\left(0^{\circ}, 0\right.$ $\mathrm{mm}),\left(0^{\circ}, \pm 30 \mathrm{~mm}\right),\left(-20^{\circ}, \pm 30\right.$ and $\left.0 \mathrm{~mm}\right),\left(30^{\circ}, \pm 30\right.$ and $\left.0 \mathrm{~mm}\right)$.

- Thickness of the bi-layers. This variation only applied to the constant d-spacing coatings.

These requirements resulted in the selection of samples listed in table 2. With the exception of the fused sillica samples, seso1 and seso2, all sample substrates are from Schott Glass of the type AF 45 which is a modified borosilicate glass with a high content of $\mathrm{BaO}$ and $\mathrm{AL}_{2} \mathrm{O}_{3}$. FLAT1 is $10 \mathrm{~cm}$ by $10 \mathrm{~cm}, 0.3 \mathrm{~mm}$ thick sheets of $\mathrm{AF}$ 45 before slumping, while the rest have been slumped in the procedure followed by all HEFT mirrors. Within the given radius requirements samples were chosen randomly from the available specimens, and likewise the flight graded samples were randomly chosen from an actual batch of flight mirrors coated for HEFT. It is therefore reasonable to assume, that these samples are fair representations of a typical HEFT mirror.

The values of $\Gamma=d_{\mathrm{W}} / d_{\mathrm{tot}}, \sigma$ and $d$ listed in table 2 are the averages taken across the 9 points and the assigned errors is the variation across these 9 points. The recipes given for the flight graded samples, are the same recipes used for the first HEFT flight module (HEFT1) and are given in table 1. The final column of table 2 is the average roughness that was measured from the substrates before coating.

The samples seso1 and seso2 of super polished flats are from the Société Européene de Systemes Optiques (SESO) and have with optical micro-map measurements been determined to have a roughness better than $2 \AA$. Measurements at $8 \mathrm{keV}$ of the same surfaces proved them to have an x-ray roughness of $3.3 \AA$ and they were chosen as reference samples.

\section{4. $8 \mathrm{KEV} \mathrm{MEASUREMENTS} \mathrm{AT} \mathrm{DSRI}$}

\subsection{X-ray Measurements}

All measurements at $8 \mathrm{keV}$ were performed at the Danish Space Research Institute using the instrumental arrangement sketched in figure 2. The source is a $\mathrm{Cu}$ rotating anode operated at $6-\mathrm{kW}$ and the geometry of the anode and cathode filament yields a beam size of $1 \times 1 \mathrm{~mm}$. Slits 1 and 2 were for normal use all set to a horizontal width of $0.2 \mathrm{~mm}$ and height $5 \mathrm{~mm}$ causing an in-plane divergence of the beam over the distance

Table 2. $8 \mathrm{keV}$ data

\begin{tabular}{|l|l|l|c|c|c|c|c|}
\hline Sample & Substrate & Type of Coating & $\mathrm{N}_{\text {bilayers }}$ & d-spacing / A & $\Gamma$ & $\sigma / \AA$ & $\sigma_{\text {sub }} / \AA$ \\
\hline L12 & curved & constant d & 10 & $65 \pm 2$ & 0.37 & $3.55 \pm 0.1$ & 4.0 \\
L32 & curved & constant d & 10 & $46.5 \pm 0.5$ & 0.37 & 3.55 & $3.1 \pm 0.5$ \\
L72-1 & curved & constant d & 10 & $113.25 \pm 4$ & 0.31 & $4.7 \pm 0.1$ & $4.0 \pm 0.4$ \\
seso1 & fused sillica flat & constant d & 10 & 71.5 & 0.36 & 3.1 & 3.3 \\
FLAT1 & AF45 flat & constant d & 10 & $31 \pm 0.2$ & 0.42 & 3.5 & 3.6 \\
seso2 & fused sillica flat & flight & recipe 5 & - & - & 3.3 & 3.3 \\
L03 & curved & flight & recipe 1 & - & - & 3.25 & - \\
L29 & curved & flight & recipe 5 & - & - & $3.45 \pm 0.15$ & - \\
L72-2 & curved & flight & - & - & $3.7 \pm 0.3$ & - \\
\hline
\end{tabular}




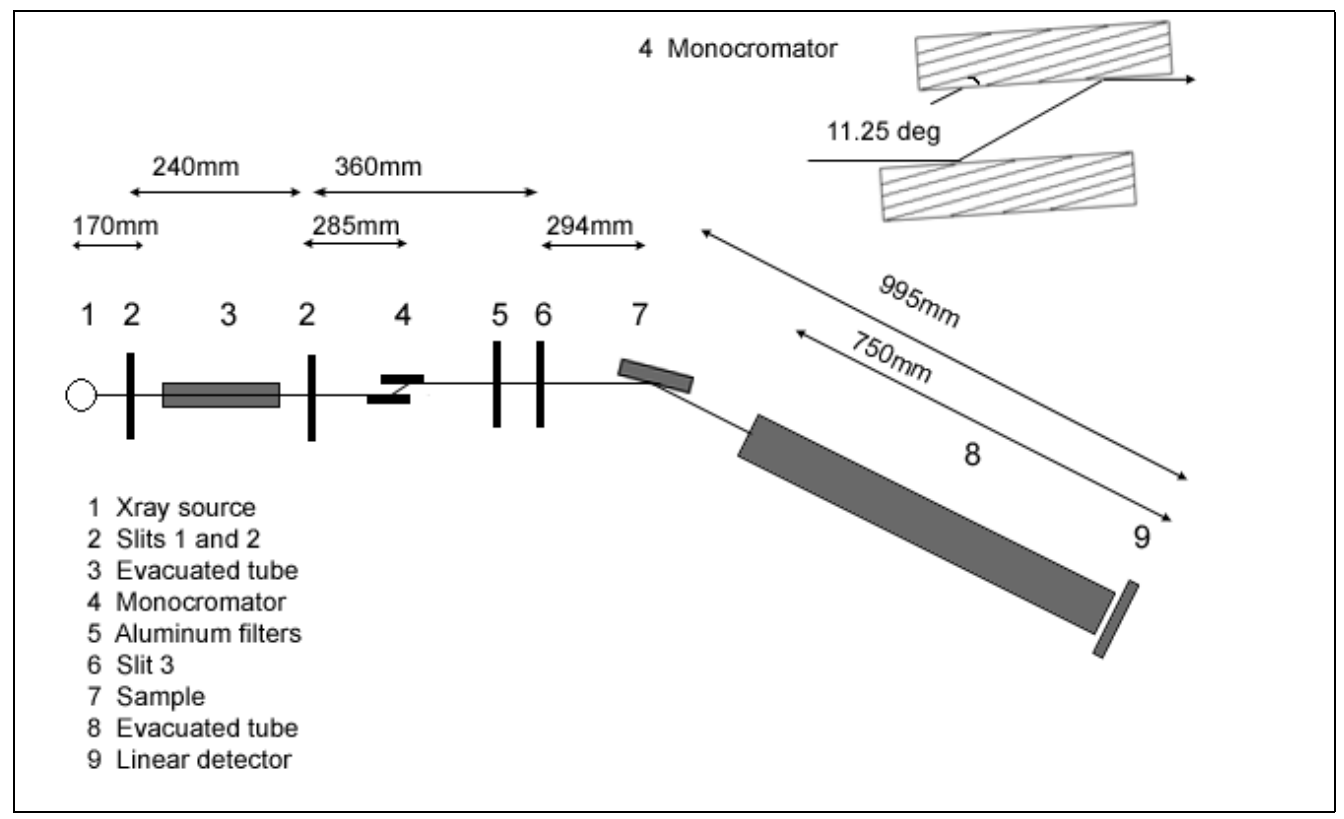

Figure 2. The instrumental arrangement at the DSRI

between the slits of $\Delta \theta_{\text {slit }}=1.146^{\prime}$. Because of natural line broadening of the $\mathrm{K} \alpha_{1}$ line, a reflection off the crystal will cause a divergence in the angle according to the Bragg formula

$$
\frac{d \lambda}{d \theta}=\frac{1}{n} 2 d \cos \left(\theta_{B}\right) \Longrightarrow \Delta \theta=\frac{\Delta \lambda}{\lambda_{K \alpha_{1}}} \tan \left(\theta_{B, K \alpha_{1}}\right)
$$

Since $\lambda_{K \alpha_{1}}=1.5404 \AA$ and $\theta_{B, K \alpha_{1}}=13.641^{\circ}$ the divergence in angle becomes $\Delta \theta=0.334^{\prime}$.

The monochromator was an asymmetrically cut Ge(111) crystal of a two bounce design with planes aligned $11.25^{\circ}$ to the surface. The two bounce non-dispersive design allowed the direction of propagation to be retained and the contraction at the first reflection to be reversed by the second reflection. The Darwin width of a symmetric $\mathrm{Ge}(111)$ crystal is $\zeta_{D}=15.3^{\prime \prime}$ and the asymmetry factor $b=10.09$ which increases the angular divergence to ${ }^{8}$

$$
\Delta \theta_{\text {mono }}=\sqrt{b} \zeta_{D}=49^{\prime \prime} .
$$

Convolving this with the value obtained from natural broadening, the beam after passage of the monochromator has undergone a total divergence of $\Delta \theta_{\text {mono }} \simeq 53^{\prime \prime}$. Comparing this with the value $\Delta \theta_{\text {slit }}=1.146^{\prime}$ the divergence is determined by the monochomator as it is smaller than the divergence from the slits. The divergence of the arrangement before the sample is thus $\Delta \theta_{\text {in }}=53^{\prime \prime}$. Futher updates will include a line-focus of width $0.2 \mathrm{~mm}$ comparable to the width of slit 1 and 2 .

A Position Sensitive Detector was used with a positional resolution of $0.3 \mathrm{~mm}$ or 1.04 ' as seen over the sampledetector distance. A window of rejection across the detector could be used as a virtual detector slit. For all specular reflectivity scans the width of the window was $4.82 \mathrm{~mm}$, while it was $1.44 \mathrm{~mm}$ for the transverse scans. The entire length of the detector was needed for the $2 \mathrm{~d}$-map which covered the scattering angles from -2.5 to 2.5 deg.

The data sets cover a dynamical range of about 7 orders of magnitudes. Figure 3 shows a 2-dimensional map in the $\theta_{\text {inci }}-\theta_{\text {scat }}$ space, where $\theta_{\text {scat }}$ is the deviation from the specular condition, of a $N=10$ constant-d coating and three examples of how this map may be cut. The map clearly shows the specular reflectivity and its diffuse component dominated by the scattering streaks originating from the Bragg peaks. At the tip of the second scattering streak part of the Yoneda line can be seen, which is where the electrical field reaches a maximum when either the incidence or exit angle is equal to the critical angle of total external reflection. 
The two lower plots have been extracted straightforwardly by laying down cuts along the specular condition, and the scattering streaks associated with the second order Bragg peak and summing across a set number of detector channels. Singular specular reflectivity scans and transverse scans are indistinguishable from the above cuts. The 'off-set' specular scan has not been depicted. Because of the streaks it looks similar to the specular merely with a lower intensity and shifted slightly.

A map from a flight coated sample does not show the same kind of distinct structure and figure 7 of a flight coated mirror clearly shows how the scatter is distributed more diffusely.
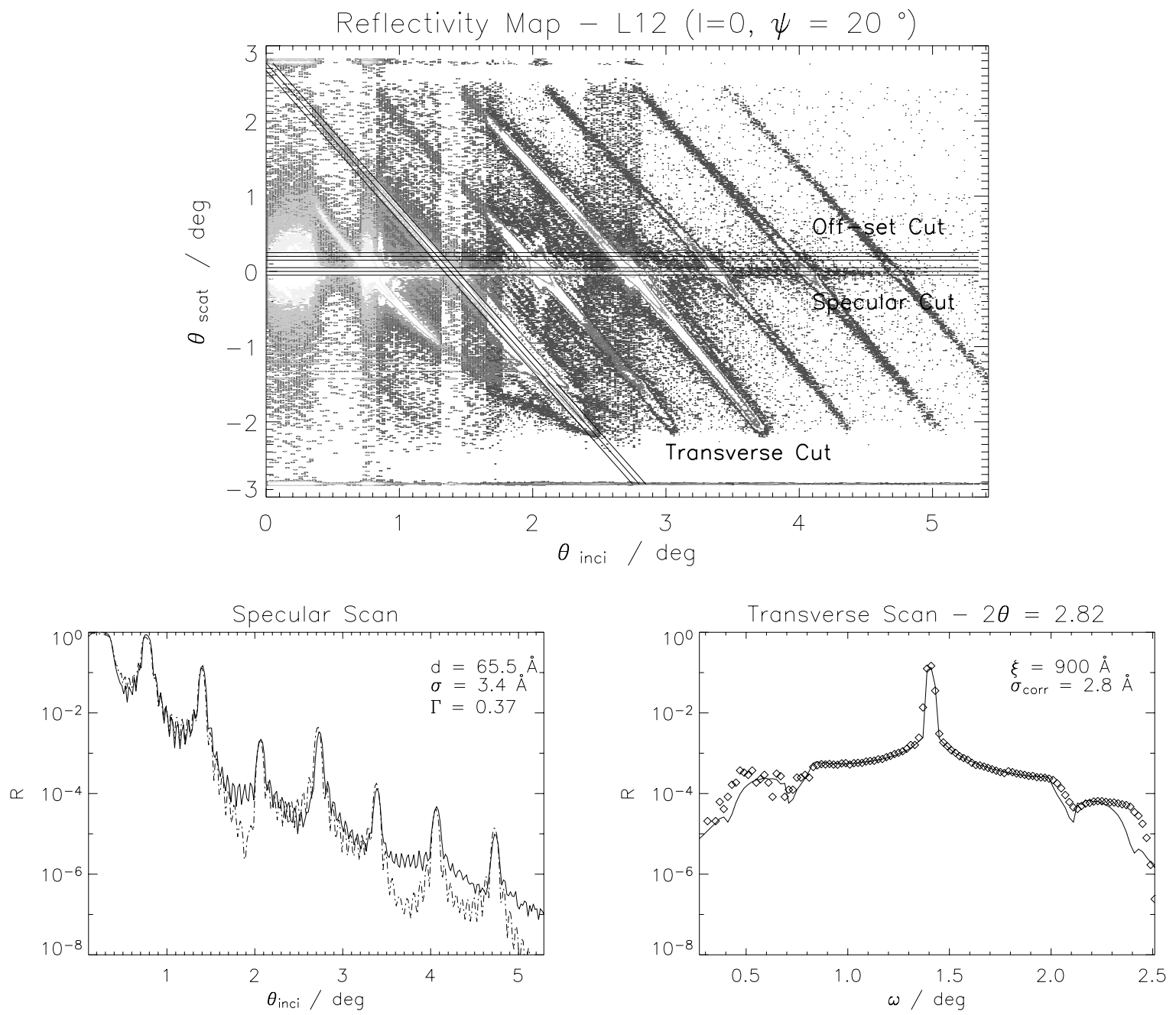

Figure 3. Diffuse reflectivity map. The scattering angle, $\theta_{\text {scat }}$ is simply the deviation in angle from the specular condition. The horizontal stripes in the background are the ghostly presence of filters, which arise due to the fact that statistically there were more non-zero events at smaller grazing angles, where the count time was shorter, than at greater angles. For the specular scan the solid line represents the data while the dash-dotted is the Nèvot-Croce reflectivity. The specular scan has been corrected with the off-set scan. The off-set scan has not been shown, but has the same shape as the specular, although with less intensity. Also the width of the peaks, are comparable to the specular and so $\sigma_{\text {ucorr }}$ can be assumed negligible. For the Transverse scan the solid line is the correlated model while the diamonds are the data. 

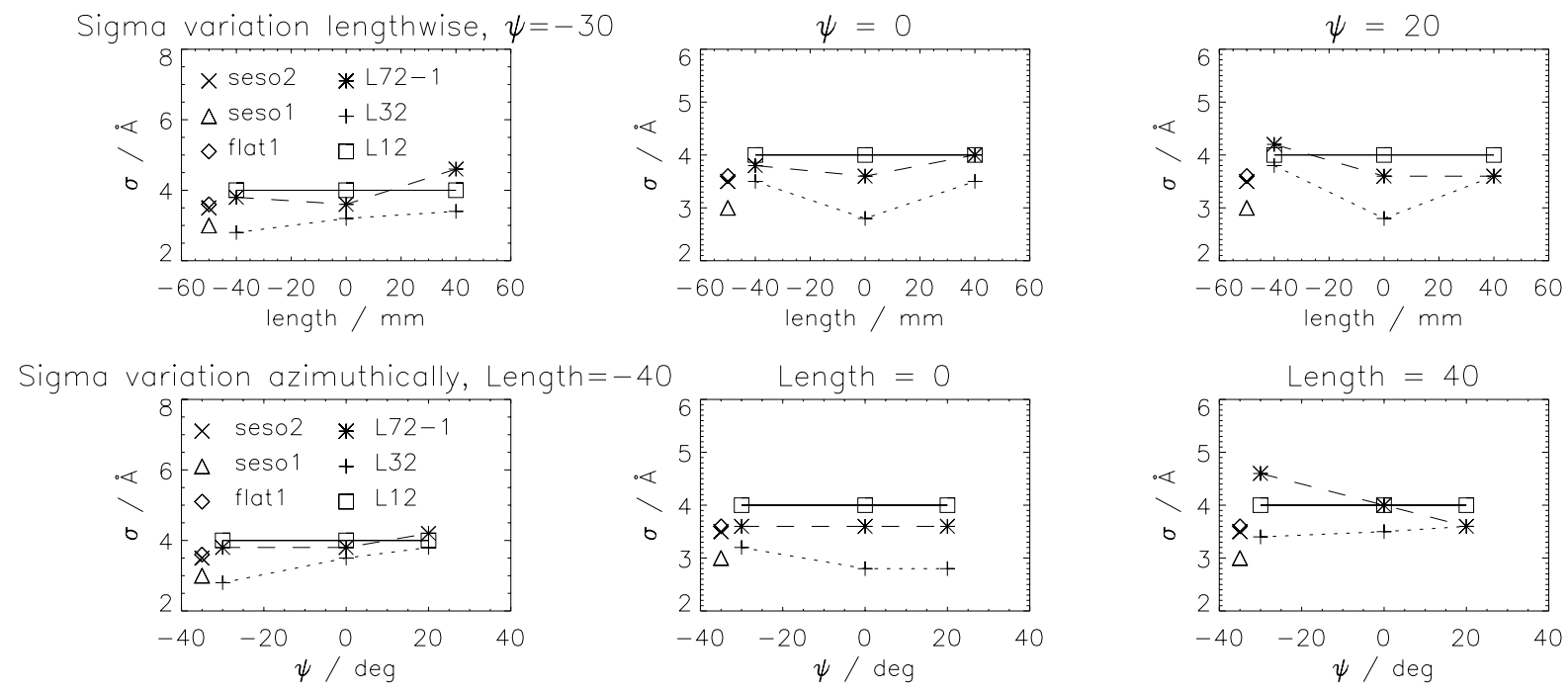

Figure 4. Roughness variation for substrates at $8 \mathrm{keV}$. The top panel shows the variation in rms roughness $\sigma$ lengthwise at azimuthal positions (from left to right): $-30^{\circ}, 0^{\circ},+20^{\circ}$. The bottom panel shows the same variation in azimuthal direction at lengths: $-40,0,40 \mathrm{~mm}$. The singular points of seso1, seso2 and FLAT1 are measured on the center spot.

\subsection{Data Analysis}

All measurements were background corrected and normalized with the direct beam taken before each measurement. The specular scan was also corrected with the 'off-set' specular scan. No other corrections were performed on the data before fitting, and instead, the models themselves were designed to take into account the surface illumination effect, which produces the characteristic asymmetry in the transverse scans due to the fact that at smaller grazing incidences a larger area of the surface is being illuminated than at greater incidences. Data extracted from the $2 \mathrm{~d}$-map were corrected with the width of the cut, which was comparable to the rejection window set during the taking of actual $(\theta, 2 \theta)$ and $\omega$ scans.

\subsubsection{Substrates}

Figure 4 shows the variation of the roughness, $\sigma$, across the surface in the azimuthal direction and lengthwise. At first glance there appears to be no correlation. However, there are a few systematic reoccurrences that are worth noting. Firstly, in general both profiles going through the point $(\psi, l)=\left(0^{\circ}, 0 \mathrm{~mm}\right)$ have a smoother and lower roughness than at the corners. Secondly, there appears for larger radii to be a greater variation of the roughness across the surface. Both things may be explained by the manner in which the mirrors are thermally slumped, but this has as yet not been thoroughly investigated. The flat reference sample FLAT1 has been measured at three points, and the values are distributed about the average of the curved sample set. There is therefore no reason to suspect that the slumping procedure causes any significant changes to the overall roughness.

\subsubsection{Constant d-spacing}

Figure 5 shows the roughness variation across the surface of the $\mathrm{N}=10$ constant-d coated mirrors. Comparing this with figure 4, shows that the variation in both directions appears to be more even. Figure 6 gives the d-spacing for the same two samples normalized to the central measurement point $(\psi, l)=\left(0^{\circ}, 0 \mathrm{~mm}\right)$ and off-set by 0.1 . It can be seen that the uniformity of the coating only varies with a few percent and does not seem to be correlated with the roughness. This suggests that to some extent a $\mathrm{W} / \mathrm{Si}$ coating is able to even out the surface roughness variations from the substrate.

Figure 3 shows an example of a transverse $\omega$ scan and its accompanying fit assuming a correlated roughness. The two lower plots show the cuts indicated in the map and their fitting parameters. As mentioned the specular reflectivity first had to be corrected with the 'off-set' specular scan to obtain the true $\sigma$, which was found to be 

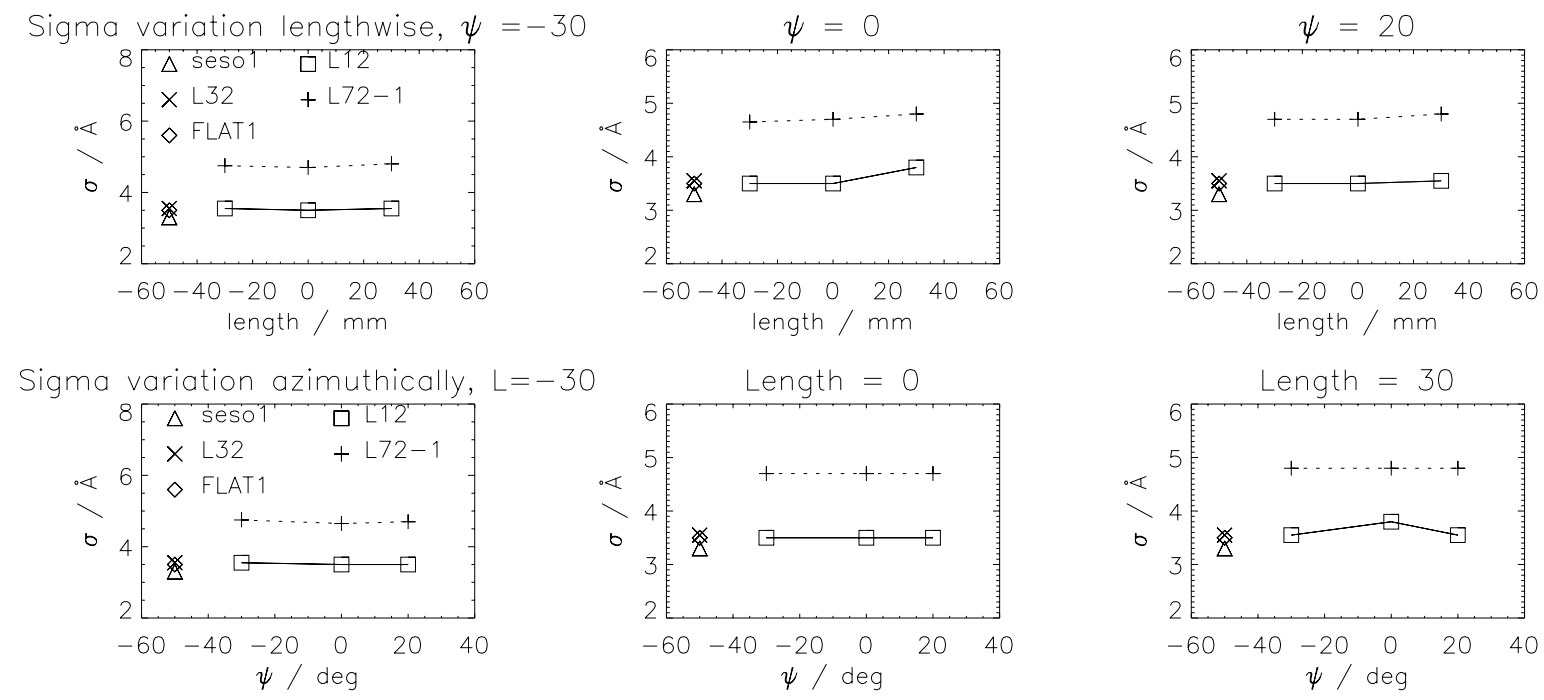

Figure 5. Roughness variation for $\mathrm{N}=10$ at $8 \mathrm{keV}$. The top panel shows the variation in rms roughness $\sigma$ lengthwise at azimuthal positions (from left to right): $-30^{\circ}, 0^{\circ},+20^{\circ}$. The bottom panel shows the same variation in azimuthal direction at lengths: $-40,0,40 \mathrm{~mm}$. The singular points of seso1 and FLAT1 are measured on the center spot.
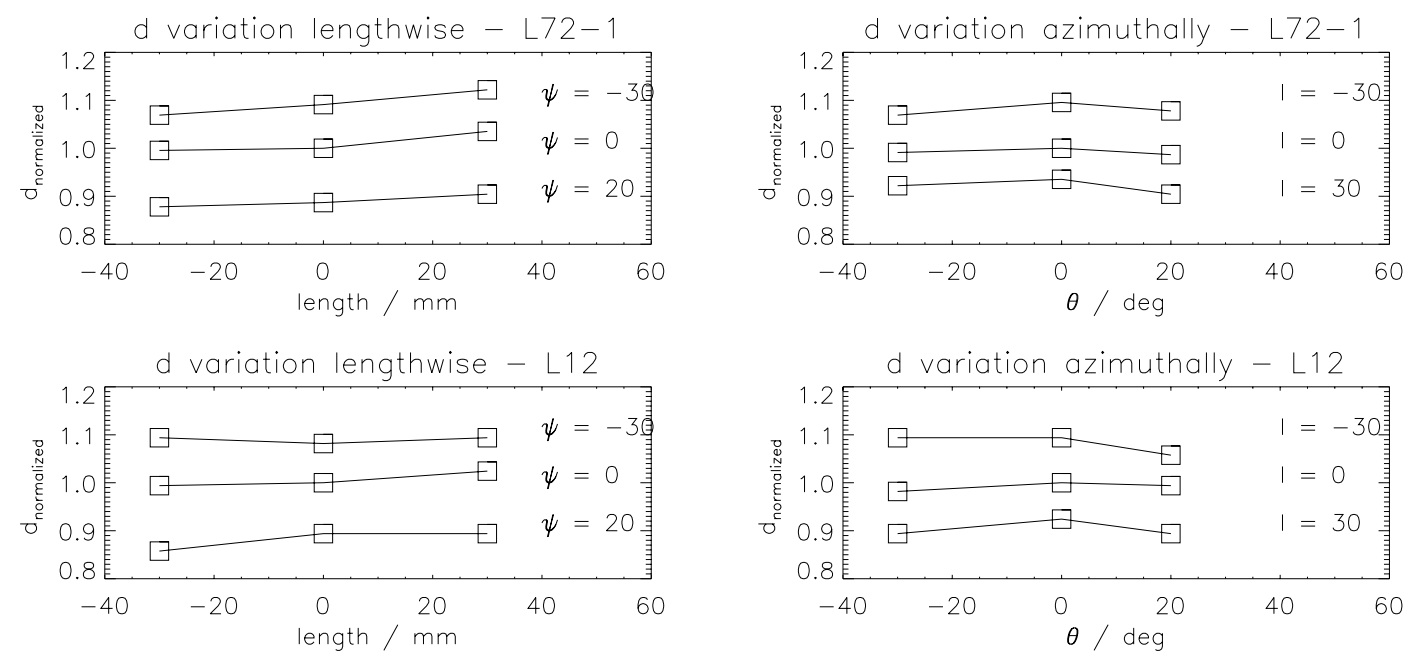

Figure 6. Variation of d-spacing. The thickness has been normalized with the value of the central measurement point $(\theta, l)=\left(0^{\circ}, 0 \mathrm{~mm}\right)$ and the data sets of constant length or angle have been offset 0.1

$\sigma=3.5 \AA$ at that particular point. The average of the entire sample was $\sigma_{\text {ave }}=3.55 \pm 0.1 \AA$. The transverse scan taken along the second Bragg peak clearly exhibits the interference humps at about $0.6^{\circ}$ and $2.3^{\circ}$ of the first and third Bragg peaks. The roughness of the perfectly correlated roughness fit was found to be $\sigma_{\text {corr }}=2.8$ $\AA$ and the correlation length $\xi=900 \pm 100 \AA$. The fractal exponent was $\mathrm{h}=0.2$, which implies that the type of roughness is of the jagged, relatively high frequency type.

Table 3 shows the results of the transverse measurements. For all three curved samples transverse measurements for at least two different Bragg peaks have been included. It clearly indicates the reoccurrence of fit parameters from point to point on a sample, and also from sample to sample. As expected the sample seso1 falls outside this, and not surprisingly it has a shorter correlation length, which is in good agreement with our expectations that it is a more highly polished sample than the mirrors. What is especially interesting for this sample is, that while the roughness is lower the interfacial grading is comparable to that of the curved mirrors. 


\begin{tabular}{|c|c|c|c|c|c|c|c|}
\hline Sample & Position & $2 \theta / \operatorname{deg}$ & $\xi(\AA \pm 100)$ & $\mathrm{h}$ & $\sigma_{\text {corr }}(\AA \pm 0.1)$ & $\sigma_{d}(\AA \pm 0.1)$ & $\sqrt{\sigma_{\text {corr }}^{2}+\sigma_{d}^{2}}(\AA \pm 0.15)$ \\
\hline L72-1 & $-20^{\circ}, 30 \mathrm{~mm}$ & 1.70 & 900 & 0.2 & 3.5 & 3.7 & 5.1 \\
\hline- & $-30^{\circ},-30 \mathrm{~mm}$ & 1.79 & 900 & 0.2 & 3.5 & 3.7 & 5.1 \\
\hline - & $20^{\circ},-30 \mathrm{~mm}$ & 1.69 & 900 & 0.3 & 3.5 & 3.6 & 5 \\
\hline - & $0^{\circ}, 0 \mathrm{~mm}$ & 3.96 & 900 & 0.2 & 2.6 & 4.0 & 4.8 \\
\hline- & $0^{\circ}, 0 \mathrm{~mm}$ & 6.27 & 900 & 0.2 & 2.6 & 4.0 & 4.8 \\
\hline L32 & $0^{\circ}, 0 \mathrm{~mm}$ & 2.06 & 900 & 0.2 & 2.3 & 2.7 & 3.5 \\
\hline- & $0^{\circ}, 0 \mathrm{~mm}$ & 3.81 & 900 & 0.2 & 2.3 & 2.7 & 3.5 \\
\hline- & $20^{\circ}, 0 \mathrm{~mm}$ & 2.1 & 900 & 0.2 & 2.5 & 2.5 & 3.5 \\
\hline- & $20^{\circ}, 0 \mathrm{~mm}$ & 3.88 & 900 & 0.2 & 2.5 & 3.0 & 3.9 \\
\hline - & $20^{\circ},-25 \mathrm{~mm}$ & 2.1 & 900 & 0.2 & 2.5 & 2.5 & 3.5 \\
\hline - & $20^{\circ},-25 \mathrm{~mm}$ & 3.88 & 500 & 0.2 & 2.3 & 3.0 & 3.8 \\
\hline - & $-30^{\circ},-25 \mathrm{~mm}$ & 2.1 & 500 & 0.2 & 2.5 & 2.7 & 3.7 \\
\hline- & $-30^{\circ},-25 \mathrm{~mm}$ & 3.88 & 500 & 0.2 & 2.5 & 2.7 & 3.7 \\
\hline L12 & $0^{\circ}, 0 \mathrm{~mm}$ & 1.58 & 900 & 0.2 & 2.5 & 2.5 & 3.5 \\
\hline- & $0^{\circ}, 0 \mathrm{~mm}$ & 2.84 & 900 & 0.2 & 2.5 & 2.0 & 3.2 \\
\hline- & $0^{\circ}, 0 \mathrm{~mm}$ & 4.13 & $500<$ & 0.2 & 2.5 & 2.5 & 3.5 \\
\hline - & $20^{\circ}, 0 \mathrm{~mm}$ & 1.57 & 900 & 0.2 & 2.8 & 2.0 & 3.4 \\
\hline - & $20^{\circ}, 0 \mathrm{~mm}$ & 2.82 & 900 & 0.2 & 2.8 & 2.0 & 3.4 \\
\hline - & $20^{\circ}, 0 \mathrm{~mm}$ & 4.12 & 900 & 0.2 & 2.7 & 2.5 & 3.7 \\
\hline FLAT1 & $0^{\circ}, 0 \mathrm{~mm}$ & 2.92 & 900 & 0.2 & 2.5 & 2.8 & 3.8 \\
\hline- & $0^{\circ}, 0 \mathrm{~mm}$ & 5.72 & 900 & 0.2 & 2.5 & 2.8 & 3.8 \\
\hline sesol & center & 1.44 & 300 & 0.2 & 1.5 & 3.0 & 3.4 \\
\hline- & center & 2.6 & 300 & 0.2 & 1.7 & 2.8 & 3.2 \\
\hline _- & center & 5 & 300 & 0.2 & 1.5 & 3.0 & 3.4 \\
\hline
\end{tabular}

Table 3. Transverse measurements at $8 \mathrm{keV}$.

This points to the diffusion being strongly related to the deposited materials and not to the type of substrate.

A Comparison of the correlated roughness from sample to sample indicates that the overall roughness is higher for larger radii mirrors and gradually decreasing for smaller radii. The effect of the graded interface is to attenuate the intensity all along the transverse scans, and not redistribute it like real roughness. The attenuation fractor is independent of graze angle, so while the graze angles are small and the reflected intensity is high, the diffusion has only a weak effect and most low order Bragg peaks could be fitted without taking grading into account. However as the reflectivity drops with graze angle, the attenuation from diffusion grows more dominant and eventaully becomes the main contributor. ${ }^{9}$ The interfacial diffusion width derived from the higher orders is therefore more exactly determined, while at low orders although its inclusion does not contradict the fitted model neither is it absolutely required. The final column of table 3 shows the squared sum of the two roughness types. Though the values are a little on the high side, taking the uncertainties into account, they are not unreasonably far from the overall interfacial width obtained by fitting the specular reflectivity curve given in table 2 .

\subsubsection{Flight graded}

On the left side of figure 7 is a 2-dimensional map of the flight graded sample seso2. As can be seen in this figure, this kind of map lacks the structure strongly dominating the constant-d coatings. Ghostly streaks obeying the Bragg condition can still be seen where the electrical field finds a local maximum, and the specular shape, given as the solid line in the right plot, is partly replicated in the diffuse component (dash-dotted). On the right side of figure 7 the intensities for the specular (solid) line, and diffuse component (dash-dotted) are shown. It is apparent that for higher grazing incidences the specular reflectivity approaches the diffuse component since the efficiency of the multilayers to coherently interfere is lost, while the power of the scatter remains much the same. For the HEFT telescope, where only angles up to $1^{\circ}$ are relevant, 5 percent of the intensity at most is lost to scatter. 

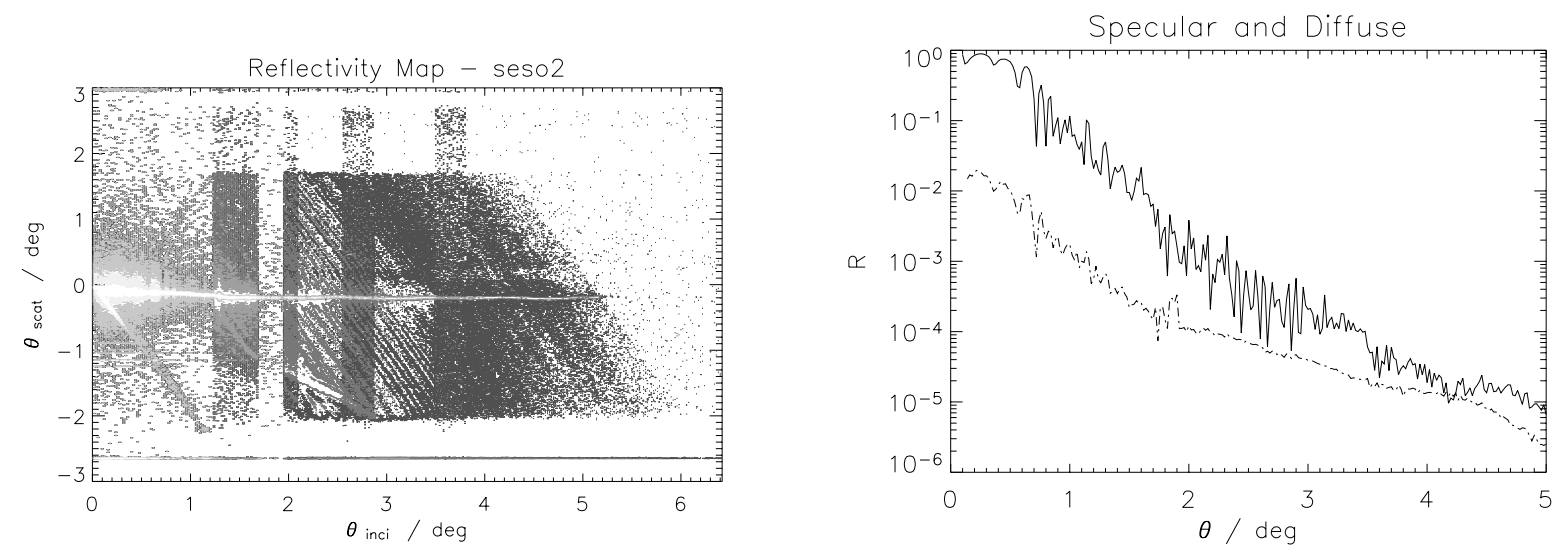

Figure 7. Left: 2-D map of a flight graded sample. Transverse streaks arise at the points where the electrical field has a maximum, i.e. at the peaks of the specular reflectivity. The streak at negative scattering angles and at grazing incidence of about 2 comes from the Yoneda condition where the exit angle is equal to the critical. The strong streak out from 0 to negative angles is due to the finite length of the sample and is the radiation which 'misses' the sample. The streak at positive angles is suspected to be due to facetted edges of the sample. Right: Solid line is the specular reflectivity while the dash-dotted is the diffuse component summed across the interval $\theta_{\text {scat }}=\left(0.2^{\circ}, 1.45^{\circ}\right)$.

Figure 8 shows the sample $L 03$ at 8 and $40 \mathrm{keV}$. The specular reflectivity scans taken at different positions are similar and the roughness, $\sigma$, the same. This shows that the flight coatings performs as expected at all energies and suggest, as it did for the constant-d coatings, that the coating is uniform and can smooth out some of the roughness fluctuations in the substrate.

\section{HIGH ENERGY MEASUREMENTS AT ESRF}

High energy measurements were performed on a few selected samples at the European Synchrotron Radiation Facility on the optics beamline BM05. The instrumental arrangement used a double bounce $\mathrm{Si}(111)$ monochromator and the high degree of collimation of the synchrotron beam provided an in plane divergence of 1". The detector used was as a simple pin diode and the detector window was set by a tungsten slit. The energy range of the monochromator lies from 15 to $70 \mathrm{keV}$ and it could be operated in different modes, one of which was to make an energy scan of a sample with the angle of incidence held fixed.

The samples measured are given in table 4 . On the first three samples specular reflectivity and transverse $\omega$-scans were performed at position $(\psi, l)=\left(0^{\circ}, 0 \mathrm{~mm}\right)$, while the final sample, L03 was mapped in all 9 points at energies 40 and $60 \mathrm{keV}$. Figure 8 shows all 9 specular reflectivity curves at $40 \mathrm{keV}$ for L03 offset for viewing purposes by a factor of 10 . The roughness is about the same for all, $\sigma=3.25 \pm 0.1$, as it also was for the sample at $60 \mathrm{keV}$. The coating thus retains its uniformity at all depths probed by the soft and hard x-rays.

Figure 9 shows specular reflectivities at $40 \mathrm{keV}$ for the samples L72-1 and seso1. The fit parameters are close to the ones obtained at $8 \mathrm{keV}$ as expected.

The final type of scan that was produced at high energies, was the energy scan. Figure 10 shows a series of energy scans that were performed on the two-bounce optic HEFT1. They are single reflectance values and have

\begin{tabular}{|l|c|l|c|c|c|}
\hline Sample & Energy $(\mathrm{keV})$ & Measurement type & $\sigma$ & $\Gamma$ & $\mathrm{d}$ \\
\hline seso1 & 40 & single & 3.25 & 0.36 & 72.25 \\
L32 & 40 & single & 3.5 & 0.3 & 47 \\
L72-1 & 40 & single & 4.0 & 0.315 & 113.5 \\
L03 & 40 & mapped & $3.25 \pm 0.1$ & - & - \\
L03 & 60 & mapped & $3.25 \pm 0.1$ & - & - \\
\hline
\end{tabular}

Table 4. Measurements obtained at the ESRF at $40 \mathrm{keV}$. 

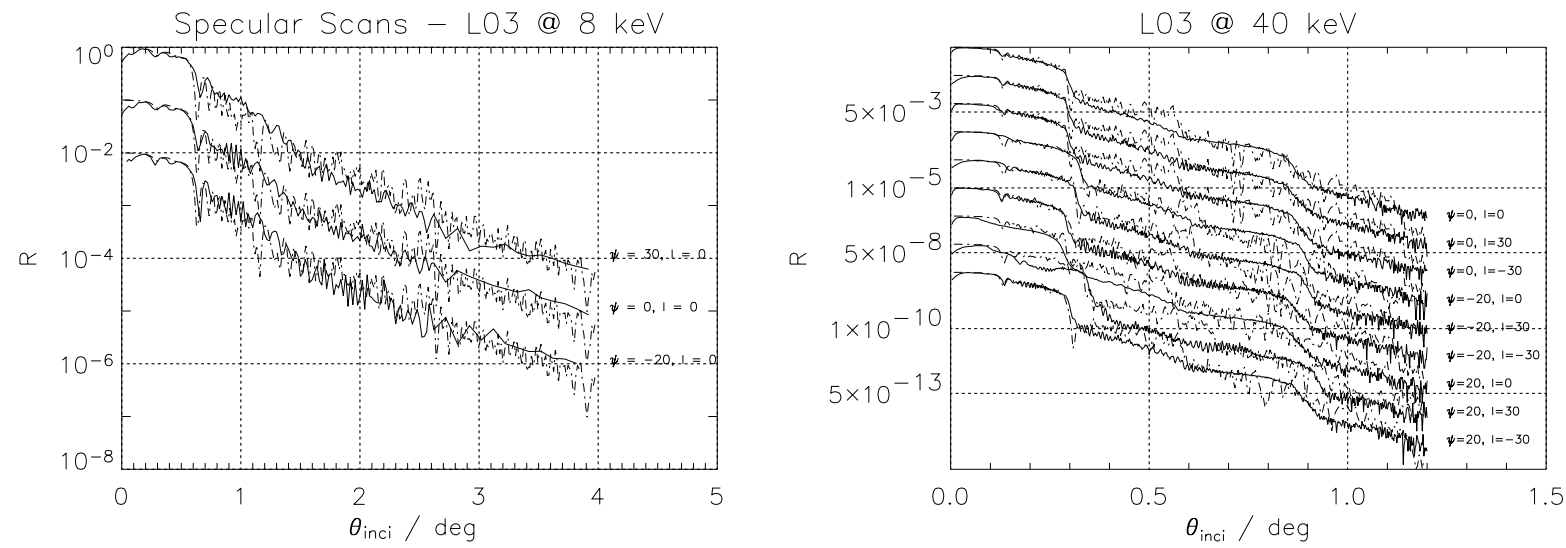

Figure 8. Sample L03 mapped out a 8 and $40 \mathrm{keV}$. Graphs are offset by a factor of 10 and the dashed underlying graphs are the Nevot Croce fits. All fits have a rms roughness, $\sigma=3.25 \AA$.
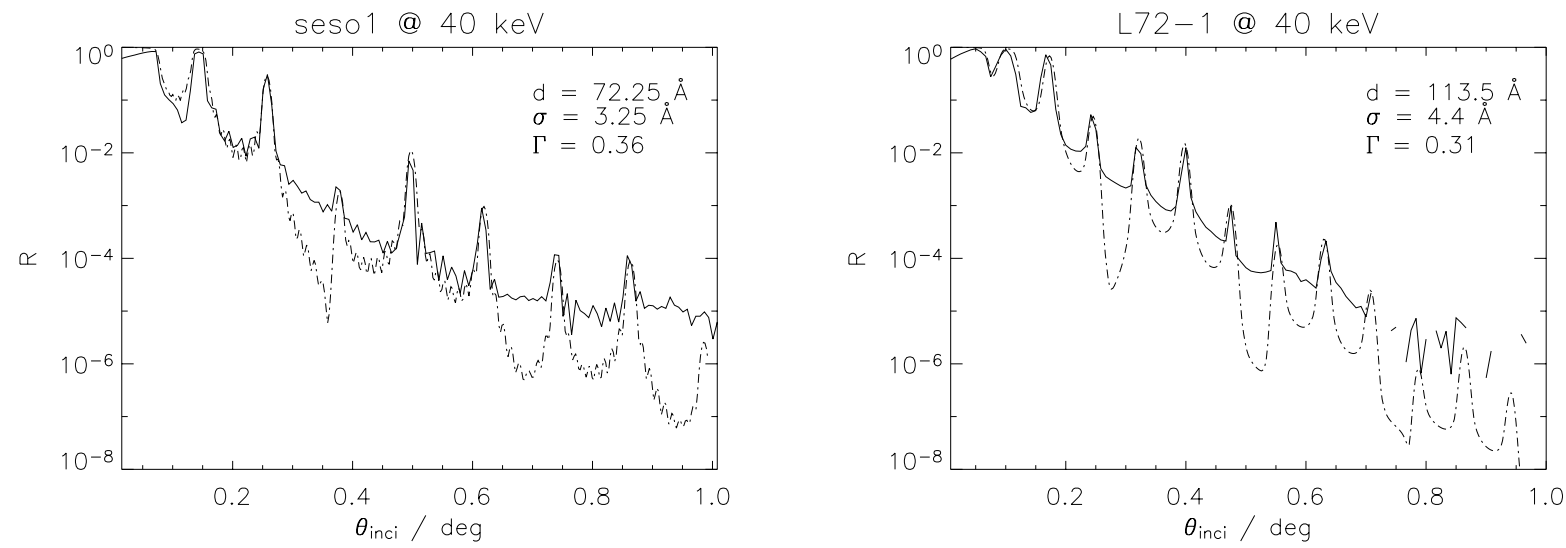

Figure 9. Specular reflectivity at $40 \mathrm{keV}$. Solid lines are the experimental data and the dash-dotted the Névot-Croce reflectivity.

been corrected with the throughput values assigned to the individual telescope shells determined at $8 \mathrm{keV}$. All of them follow the energy response predicted by their coating recipe and predictably drop at $69 \mathrm{keV}$ where the tungsten absorption edge is located. The thick diamond at $8 \mathrm{keV}$ is a measurement point from $8 \mathrm{keV}$ data.

\section{SUMMARY AND DISCUSSION}

In section 3.1 we set up four parameters that we wished to investigate. These were: Energy, radius of the mirrors, uniformity of the mirror surfaces and thickness of the bilayers. To these four we might add coating type and sample type as well. All in all we had a large parameter space to work with and a brief summary is best given by following the evolution of a mirror from before slumping to after is has been coated.

Starting with the flat AF 45 glass substrate, it was expectedly found to be quite uniform and there is some evidence that slumping does introduce a small increase in the roughness variation across the surface, especially at the edges and corners. When a coating is applied, constant or flight, the roughness is found to be correlated and the variation from the substrate to be slightly smoothed out. There is some variation in bilayer thickness across the surface which in percent of the total bilayer thickness is about the same for all samples, but it does not seem to be correlated to the roughness variation. The rms roughness also has a small increase for larger radii samples and was discovered to have a large contribution from interfacial diffusion to the total interfacial width. The correlation length was determined to lie in the range of 500-900 $\AA$ for all the AF 45 samples. The samples 

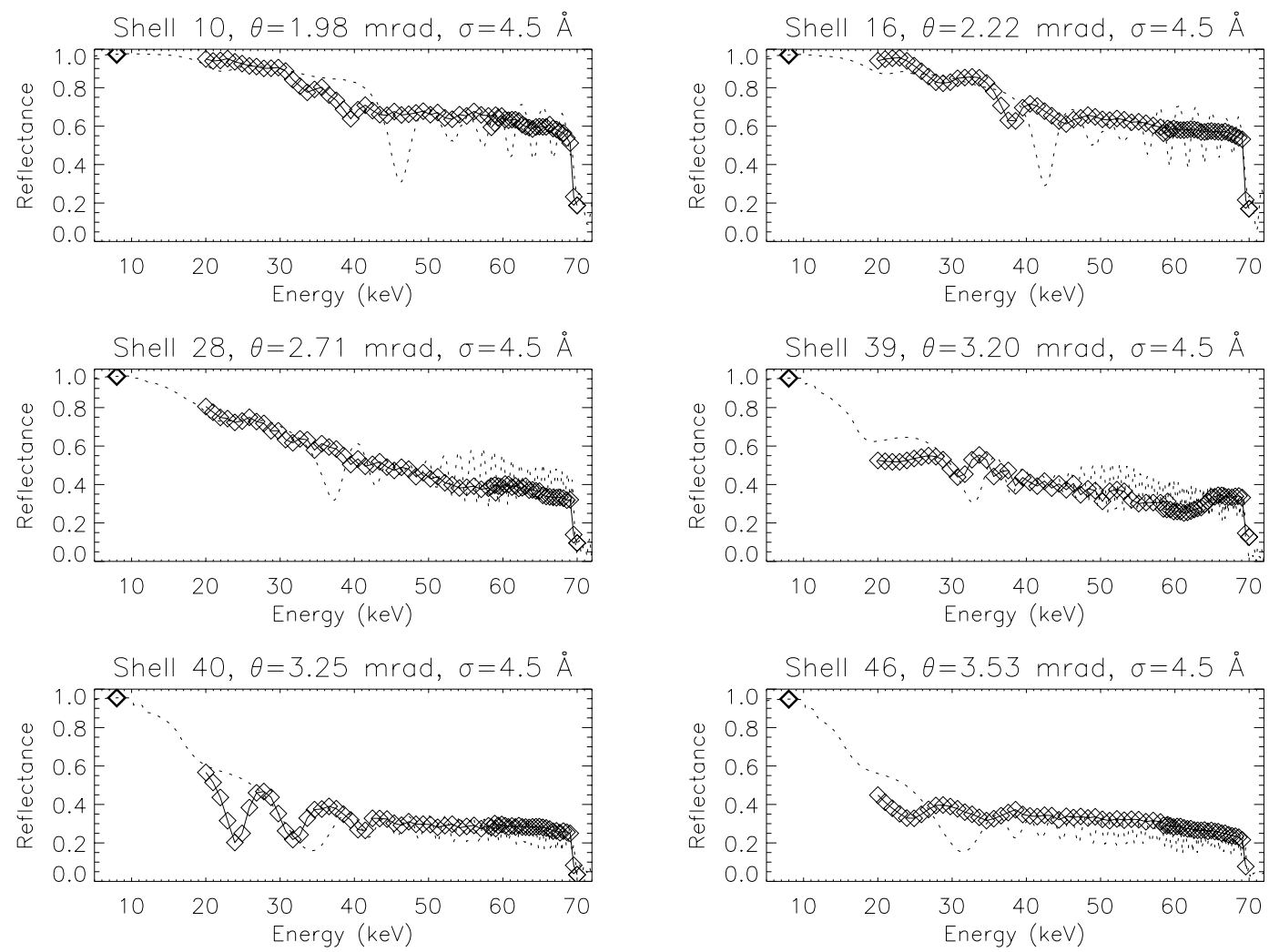

Figure 10. Energy scans from $20 \mathrm{keV}$ to $70 \mathrm{keV}$. The shell labels refer to the layer so that shell 16 is a L16 mirror of HEFT1. The diamonds are the experimental data and the dotted line the throughput corrected Névot-Croce reflectance of the particular flight recipe used on that shell.

seso1 and seso2 were predictably found to have lower roughness and a shorter correlation length, but the diffuse interfacial width was comparable to the curved mirrors. The coatings performed as expected at higher energies with no noticeable changes in fitting parameters.

\section{REFERENCES}

1. M. Born and E. Wolf, Principles of Optics, Cambridge, seventh (expanded) edition ed., 1977.

2. D. G. Stearns, "The scattering of x-rays from nonideal multilayer structures," J. Appl. Phys 65, pp. 491-506, 1988 .

3. L. Névot and P. Croce Revue Phys, Appl 15, p. 761, 1980.

4. T. B. V. Holý, "Nonspecular x-ray reflection from rough multilayers," Phys. Rev. B 49, pp. 10668 - 10 676, 1994.

5. S. K. Sinha, E. B. Sirota, S. Garoff, and H. P. Stanley, "X-ray and neutron scattering from rough surfaces," Phys. Rev. B 38, pp. 2297-2311, 1988.

6. P. H. Mao, F. A. Harrison, D. L. Windt, and F. E. Christensen, "Optimization of graded multilayer designs for astronomical x-ray telescopes," Appl. optics 38, p. 4766, 1999.

7. C. P. Jensen, K. K. Madsen, C. M. H. Chen, F. E. Christensen, and E. Ziegler, "Coating of heft telescope mirrors: method and results," Proc. SPIE 4851, p. 724, 2002.

8. J. Als-Nielsen and D. McMorrow, Elements of Modern X-ray Physics, Wiley, New York, 2001.

9. M. Wormington, I. Pape, T. P. A. Hase, B. K. Tanner, and D. Bowen Phil. Mag. Letters 74, p. 211, 1996. 